Escuela de Ciencias Sociales y Humanidades, UNED, C.R.

URL: http://investiga.uned.ac.cr/revistas/index.php/espiga/index

\title{
"Signos de los tiempos" en Gaudium et Spes Redacción, hermenéutica y teología
}

\section{Juan Pablo Espinosa-Arce}

Licenciado en Educación. Profesor de Religión y Filosofía (Universidad Católica del Maule) Estudiante del Magíster en Teología Fundamental por la Pontificia Universidad Católica de Chile. Universidad Católica del Maule. Chile. jpespinosa@uc.cl

Recibido: 29 de abril, 2015 • Aceptado: 31 de mayo, $2016 \quad$ C Corregido: 15 de agosto, 2016

\section{RESUMEN}

(Find the abstracts in English and French at the end of the article)

El siguiente artículo dará cuenta de un breve estudio de carácter redaccional y hermenéutico de la categoría teológica "Signos de los tiempos" presente en la Constitución Pastoral Gaudium et Spes del Concilio Vaticano II y en las Congregaciones Generales que prepararon dicho documento. Estudiar la teología de "Signos de los tiempos" exige que el creyente pueda asumir que en la historia y en la cultura en la que vive y convive se puede hallar la presencia de Dios para provocar nuevos caminos de actuación y renovación eclesial.

Palabras clave: Signos de los tiempos, Gaudium et Spes, Congregaciones Generales, Concilio Vaticano II, historia.

\section{Introducción}

El presente artículo pretende ser un estudio de carácter redaccional y hermenéutico a la categoría teológica "Signos de los tiempos" presente en la Constitución Pastoral Gaudium et Spes ${ }^{1}$ (Literalmente "Gozos y esperanzas") del Concilio Vaticano II. Este

\section{Formato de citación según APA}

Espinoza-Arce, J.P. (2016). "Signos de los tiempos" en Gaudium et Spes. Redacción, hermenéutica y teología. Revista Espiga. Vol. XV, (32), 119-136.

\section{Formato de citación según Chicago}

Espinoza-Arce, Juan Pablo. "“Signos de los tiempos” en Gaudium et Spes. Redacción, hermenéutica y teología». Revista Espiga. XV. n 32 (2016): 119-136.

1. De ahora en adelante GS. 
trabajo abordará la historia de la redacción de un número en particular, a saber, el número 4 perteneciente a la Parte introductoria del documento final de GS. Junto con ello las relaciones, los elementos añadidos y extraídos del número ${ }^{2}$ presente en las Congregaciones Generales 105, 132, 161 y 166. Dicho número presenta la categoría teológica de los "Signos de los tiempos", la cual es uno de los conceptos fundamentales del Concilio Vaticano II.

Pensar en los "Signos de los tiempos" exige un mirar de la realidad en clave creyente, lo cual constituye una tarea necesaria y permanente para todo el Pueblo de Dios pero de manera principal para teólogos, teólogas y pastores (Cf. GS 4,11). Este discernimiento y juicio sobre las realidades temporales y sobre la historia humana en la que se hace presente la Historia de la Salvación, debe realizarse desde una amplitud tal de horizontes que permita hacer converger criterios hermenéuticos que pongan en relación con la teología. Esta aporta el sentido de la fe, y las ciencias filosóficas y humanas, que en el caso de Gaudium et Spes se abocan en la sociología. Ahora bien ¿qué se entenderá por "signos de los tiempos"? Definir esta categoría teológica no es tarea fácil, esto porque es una teología reciente y que depende del mismo horizonte de comprensión que se tenga de ellos ${ }^{3}$.

En este desarrollo se tendrá como horizonte el ver cómo los Padres Conciliares enfrentan un tema que ya había sido mencionado por Juan XXIII en la Constitución Apostólica Humanae Salutis (Literalmente: "Salvación de la humanidad", 25 de diciembre de 1961) con la cual se convoca al Concilio Vaticano II, y cómo lo enfrentan específicamente en el mencionado número 4 de la Constitución Pastoral. Para ello se realizará un trabajo hermenéutico de corte pragmático del texto de la Humanae Salutis de Juan XXIII y de los textos de las Congregaciones Generales 105, 132, 161 y 166 en los cuales se trabaja el desarrollo de la posterior Gaudium et Spescon el fin de vislumbrar cuál es la historia de la categoría teológica que estamos estudiando.

Desde estos textos se plantearán las siguientes preguntas ¿En cuál(es) de los texto(s) se nombra "signos de los tiempos"? ¿En relación a qué se nombra? ¿Por qué se nombra

2. En la Congregación General 105 el número que se estudiará correspondía entonces al 2. Por su parte las Congregaciones Generales 132, 161 y 166 presentaron este apartado en el número 4.

3. Así, por ejemplo, G. Bausenhart(2013) define ST como "las condiciones políticas, culturales y sociales en las cuales viven los hombres, en las cuales arriesgan su existencia humana de fe" (p.32). A. Bentué (2001) sostiene que una teología de los "Signos de los tiempos" viene a fundarse en la presencia del Espíritu Santo en los acontecimientos temporales que acontecen en la historia, para que desde ellos y "lo que acontece en la historia, producido por decisiones humanas suscitadas por determinadas tomas de conciencia, vengan de donde vengan, tiene inherente, a pesar de la ambigüedad con que pueda darse, la presencia del Espíritu de Dios, que ilumina a toda conciencia en la línea de lo que él es" (p.104). J. Noemi (2001) por su parte relaciona los "Signos de los tiempos" con una interpretación teológica del presente, un discernimiento o un juicio realizado desde la óptica creyente teniendo como marco interpretativo el Evangelio, la Tradición y el lugar del Magisterio. Pero con esto Noemi advierte que la revolución provocada por Gaudium et Spes en la que tienen lugar los ST ha enfrentado a la Iglesia con una "insuficiencia metodológica (...) la articulación de una teología de los signos de los tiempos sigue siendo todavía un programa que pareciera circunscribir una aporía metodológica insuperable" (p. 441) En estos tres ejemplos advertimos las siguientes consecuencias: a) No hay una definición aparentemente consensuada sobre lo que serían los "Signos de los Tiempos", ya que se habla de condiciones en las que viven los hombres, presencia de Dios en la historia o una teología que aún no está suficientemente desarrollada la cual presenta incluso aporías metodológicas. 
o no se deja de nombrar? ¿Se mantiene o cambia el sentido que Juan XXIII le da a los "signos de los tiempos" en los textos de las Congregaciones Generales?. Junto con ello se procederá a dar un paso más, a saber, asumir que no se puede leer el número 4 de GS de manera solitaria, sino que se debe realizar en conjunto con el número 11 del texto definitivo de la Constitución Pastoral del Concilio ${ }^{4}$. Esta puesta en diálogo de GS 4 y GS 11 permitirá la consideración de la existencia de la relación teología-sociología presente en buena parte de Gaudium et Spes. Seguido de ello se preguntará el porqué discernir los "signos de los tiempos" constituye una tarea irrenunciable para la Iglesia. Para ello, se ofrecerá un breve apartado en el que se estudiará cómo la reflexión latinoamericana, específicamente la presente en el Documento de Aparecida del Episcopado Latinoamericano y del Caribe (2007) ha abordado la teología de los "signos de los tiempos". Finalmente, y a modo de síntesis volver a formular la pregunta de qué son en definitiva los "signos de los tiempos". Para ello se extraerán algunas características presentes en los textos GS 4, 11 y 44 de manera de intentar una descripción más o menos acabada sabiendo que la teología de los "signos de los tiempos" aún no está concluida.

\section{Constitución Apostólica Humanae Salutis}

Juan XXIII en la Constitución Apostólica Humanae Salutis, por medio de la cual se convoca al Concilio Vaticano II (25 de diciembre de 1961), realiza la primera mención oficial en el contexto conciliar de la categoría teológica "signos de los tiempos". La Constitución comienza presentando una dimensión cristológica, por la cual Jesucristo es "el reparador de la salvación humana" (Juan XXIII, 1971, parágrafo $1^{5}$ ), salvación que es el centro medular del Evangelio, el cual Él encomendó a la Iglesia para que lo anunciara en todo el mundo hasta el fin de los tiempos. La Iglesia, aunque posee un origen divino, se encuentra viviendo entre los seres humanos, en esta historia, en este momento actual que pasa por diversas vicisitudes. Este momento actual exige que la Iglesia "inyecte la virtud perenne, vital y divina del Evangelio en las venas de esta comunidad humana actual que se gloría de los descubrimientos recientemente realizados en los campos técnico y científico, pero que sufre también los daños de un ordenamiento social que algunos han intentado restablecer prescindiendo de Dios" (Juan XXIII, 1971, parágrafo 3).

Este diagnóstico que el Pontífice realiza conduce finalmente a la mención de la categoría teológica que estamos revisando. Así, él sostendrá que "siguiendo los consejos

4. Por efectos metodológicos de nuestro trabajo sólo estudiaremos la historia del actual texto 4 en las Acta Synodalia. Para el tratamiento hermenéutico del número 11 nos valdremos de las traducciones que ofrecen los textos de los documentos del Concilio, en nuestro caso de la edición de la Biblioteca de Autores Cristianos de 1971. Iglesia Católica. (1971). Documentos completos del Concilio Vaticano II. Madrid: Biblioteca de Autores Cristianos.

5. Los documentos magisteriales de la Iglesia no se citan con la página sino que con el número del parágrafo. Aquí seguimos la forma tradicional de citar. 
de Cristo el Señor que nos exhorta a reconocer los signos de los tiempos (Mt 16,36), en medio de tinieblas tan sombrías, percibimos numerosos indicios que parecen auspiciar un tiempo mejor para la humanidad y para la Iglesia” (Juan XXIII, 1971, parágrafo4).

De la palabra de Juan XXIII, se extraerán las siguientes consecuencias. En primer lugar, el afirmar que el reconocimiento de la Iglesia de las realidades temporales se realiza a la luz de la fe. Los cambios profundos deben pensarse desde el Evangelio. También se concluye que el discernimiento de los "signos de los tiempos" tiene un origen cristológico (Jesús nos exhorta a hacerlo), a la vez que es un ejercicio eclesial (la Iglesia recibe este mandato).

El cómo del discernimiento no se explicita, pero sí que este ejercicio es uno de carácter teologal. Ahora bien ¿qué situaciones del mundo son descritas/diagnósticos por Juan XXIII). El Pontífice nombra: las guerras que provocan cambios en el orden espiritual a la vez que causan la aparición de nuevas ideologías; el progreso técnico el cual es un 'arma de doble filo', ya que hace que la persona cree nuevas armas usadas en su propia destrucción, pero también el progreso técnico permite que la persona desee la paz de manera que los pueblos puedan ayudarse entre sí.

En segundo lugar, se evidencia que la categoría "signos de los tiempos" tiene fundamento bíblico y es retomada por Juan XXIII en ese sentido. Juan XXIII no define explícitamente qué entiende por esta categoría, pero por la forma en que está ubicada en el texto, se puede pensar que ellos son signos del Reino de Dios (ver nota 5).

Finalmente, se sostiene que los "signos de los tiempos" a su vez tienen un componente escatológico, ya que en ellos se pueden reconocer tiempos mejores tanto para la humanidad como para la Iglesia. Así pareciera que la Iglesia se siente partícipe e íntimamente unida a la suerte del mundo. Esto es finalmente la articulación del Concilio en general y de Gaudium et Spes como caso particular.

6. La cita de Mateo 16,3 utilizada por Juan XXIII hace mención a la única vez que Jesús pronuncia esto de los signos de los tiempos: "Se acercaron los fariseos y saduceos y, para ponerle a prueba, le pidieron que les mostrase una señal del cielo. Mas él les respondió: "Al atardecer decís: "Va a hacer buen tiempo, porque el cielo tiene un rojo de fuego",y a la mañana:' Hoy habrá tormenta, porque el cielo tiene un rojo sombrío.” ¡Conque sabéis discernir el aspecto del cielo y no podéis discernir las señales de los tiempos! ¡Generación malvada y adúltera! Una señal pide y no se le dará otra señal que la señal de Jonás.” Y dejándolos, se fue". La palabra de Jesús sobre los signos de los tiempos y en este contexto del Evangelio de Mateo se relaciona con los signos del Reino. 


\section{Textos de las Congregaciones \\ Generales 105, 132, 161 y $166^{7}$}

\section{Primer esquema Congregación General \\ 105, 20 de Octubre de 1964 (AS, III, V) ${ }^{8}$}

(2) "La Iglesia escruta (discierne) los signos de los tiempos"

Para la Iglesia y para los hombres la voz y las señales de los tiempos llevan consigo la presencia de Dios. Desafortunadamente, el hombre ha dejado ausente a Dios y la invocación que el primero hace del segundo ha disminuido. Desde la luz de la fe se puede escuchar en la voz del tiempo la voz de Dios, y desde la luz de la fe se puede tomar conciencia de como el mandamiento concreto de la caridad puede enfrentar las miserias de los hombres de hoy.

Los acontecimientos de los hombres de nuestro tiempo, los conoce bien la Iglesia. Entre ellos, la sola familia humana se regocija en el crecimiento de la técnica, de la ciencia y de la tecnología.

Los hombres se comprometieron a cuidar de sí mismos, mientras algunos estaban confundidos con sentimientos de vacuidad, cansancio e incluso la desesperación con lo cual cayeron en la angustia.

La paz, que es el fruto de la caridad y de la justicia, de la verdad y de la libertad, no puede ser verdadera mientras que un número de seres humanos esté privado de la libertad; cuando se distribuyen los bienes de la tierra, otros sin embargo pasan hambre. No hay por tanto una evolución adecuada. La guerra, que es lo más trágico de todo pone en peligro (al género humano) una y otra vez.

Por esta razón, no es sorprendente que muchos hombres, aun cuando el dominio de la naturaleza los enorgullece, hoy comienzan a dudar sobre el sentido más profundo de la naturaleza humana. Con la ayuda del Espíritu Santo, el Concilio debe ser capaz de discernir las diversas cosas que le suceden a la totalidad de la familia humana. El Espíritu Santo dirige el transcurso del tiempo y renueva la faz de la tierra, Él purifica los corazones de los hombres y en medio de las vicisitudes del mundo los conduce a Cristo. Los hombres son capaces de discernir sus acciones en la civilización presente (Iglesia Católica, 1970, p. 117)

\section{Segundo esquema Congregación General 132, 21 de Septiembre de 1965 (AS, IV, I)}

\section{(4) "Esperanzas y angustias"}

Hoy vivimos una nueva era de la historia en la cual acontecen profundos y rápidos cambios. Muchos hablan de una verdadera transformación cultural. Estos cambios profundos y acelerados propios de esta 'transformación cultural' son provocados por la industria humana, con la inteligencia creativa del hombre la cual se vuelve sobre el hombre, sobre sus juicios y deseos

7. Aquí ofrecemos una traducción propia del latín al español de las Congregaciones Generales del Concilio Vaticano II.

8. Seguimos aquí la forma tradicional que la Iglesia Católica tiene para citar las Actas Synodalia, las Actas oficiales del Concilio Vaticano II. 
ya sean individuales o colectivos; sobre su forma de pensar y actuar, sobre las relaciones de los hombres entre sí (relaciones sociales).

Esta transformación y como a menudo sucede con los sucesos repentinos lleva consigo dificultades. La autoridad del hombre de nuestro tiempo se enfrenta a un ámbito extenso de aplicación. Quiere penetrar los recovecos más profundos de su corazón, aunque a menudo esto le parece incierto; cuando las leyes de la vida social las va descubriendo poco a poco, se va aferrando a ellas.

Nunca la humanidad ha tenido tanta riqueza, facultades y poderes económicos; sin embargo, una gran parte de la población se ve envuelta por el hambre y el analfabetismo. Nunca como hoy la unión entre las almas y su complementariedad se ha tornado una percepción tan vívida; sin embargo, existen fuerzas que los hacen combatir entre ellos, con la amenaza fuerte de una nueva guerra, capaz de destruir todas las cosas desde su raíz.

La mayoría de las personas de nuestra época, entre la esperanza y la angustia, y a causa de la inquietud hacen preguntas sobre el presente de estas duras condiciones. El hombre está irresistiblemente obligado a responder a los desafíos que se presentan en el curso de los acontecimientos. (Iglesia Católica, 1970, pp. 436-437)

\section{Tercer esquema Congregación General 161, 15 de Noviembre de 1965 (AS, IV, VI)}

\section{(4)"De las esperanzas y las angustias",}

Es deber y tarea de la Iglesia el escrutar los signos de los tiempos a la luz del Evangelio. Por lo tanto y usando un lenguaje inteligible para cada generación, la Iglesia debe ser capaz de responder a los perennes interrogantes sobre la vida presente y la vida futura además de la relación entre ambas. $(*)^{10}$

El mundo en que vivimos tiene expectativas y aspiraciones, las cuales son conocidas y comprendidas en su dramatismo (Comienzo del diagnóstico) En esta nueva era de la historia el género humano experimenta cambios vertiginosos. El hombre los ha creado con su inteligencia y con su creatividad. Existen deseos tanto a nivel individual como colectivo, así como a las formas de pensar y actuar en las cosas que se refieren al hombre. Se puede hablar por tanto de una verdadera transformación social que llega incluso a la vida de los creyentes (vida religiosa) (*)

Como ocurre en cualquier crisis de crecimiento, la transformación no deja de poseer dificultades. El hombre amplía su poder conocido; sin embargo, no siempre puede reducirlo a su servicio. Busca penetrar en lo más profundo de su corazón pero a menudo le parece incierto. Va descubriendo poco a poco las leyes de la vida social.

El género humano nunca había tenido tantas riquezas, bienes y poder económico, y, sin embargo, una gran parte de los habitantes del mundo sigue atormentada por el hambre y el analfabetismo. Nunca el hombre había tenido una visión tan aguda sobre el sentido de la libertad, sin embargo surgen nuevos tipos de esclavitud social y psicológica en el mundo $(*)$.Mientras el

9. Lo que aparece en cursiva, son los añadidos que se realizaron a este esquema.

10. Los párrafos que al final llevan un asterisco $\left(^{*}\right)$, son aquellos elementos añadidos respecto al Esquema anterior. Seguimos aquí el modo de estructuración de: Iglesia Católica (1970). Acta synodalia sacrosancti Concilii Oecumenici Vaticani II. Typis polyglottis Vaticanis. Ciudad del Vaticano. 
mundo es consciente de su unidad y de la dependencia que los une, está dividido en direcciones opuestas. Hay divisiones en las esferas politica, social, económica, 'racial' e ideológica y sigue el peligro de una guerra $(*)$ que destruya todo.

Si bien aumenta el intercambio de ideas, el sentido mismo de las palabras que expresan conceptos de gran importancia, tienen diferentes sentidos en las distintas ideologías $(*)$. Finalmente se busca cuidadosamente el avance temporal pero también avanzar en el crecimiento espiritual $\left({ }^{*}\right)$

Por estas situaciones complejas, a muchos de nuestros contemporáneos se les impide reconocer verdaderamente los valores perennes y ubicarlos correctamente con los recientes descubrimientos.

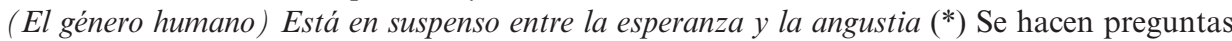
sobre el presente de las cosas. Este curso de las cosas obliga al hombre a responder (por ellos). (Iglesia Católica, 1970, pp. 425-426)

\section{Cuarto esquema Congregación General 166, 02 de Diciembre de 1965 (AS, IV, VII)}

\section{(4) "De las esperanzas y las angustias"}

Para llevar a cabo esta tarea, es responsabilidad de la Iglesia escrutar los "signos de los tiempos" a la luz del Evangelio. Por lo tanto, y usando un lenguaje inteligible y adaptándose a cada generación; debe ser capaz de responder a las perennes interrogantes sobre la vida presente, la vida futura y su relación. Para ello, es necesario conocer y comprender el mundo en que vivimos; así como sus expectativas y aspiraciones, las cuales tienen a menudo un carácter dramático. Algunas de las características más importantes del mundo de hoy pueden ser trazadas de la siguiente manera.

En esta nueva era de la historia, el género humano experimenta cambios vertiginosos. Estos cambios profundos y acelerados son provocados por la industria humana, con la inteligencia creativa de las personas, la cual se vuelve sobre ellas, sobre sus juicios y deseos, ya sean individuales o colectivos; sobre su forma de pensar y actuar, sobre las relaciones humanas entre sí. Con esto se puede hablar de una verdadera transformación social, la cual también llega a la vida religiosa.

Como ocurre en cualquier crisis de crecimiento, la transformación no deja de poseer dificultades. El hombre amplía su poder conocido; sin embargo, no siempre puede reducirlo a su servicio. Busca penetrar en lo más profundo de su corazón, pero a menudo le parece incierto. Va descubriendo poco a poco las leyes de la vida social.

El género humano nunca había tenido tantas riquezas, bienes y poder económico, y sin embargo una gran parte de los habitantes del mundo sigue atormentado por el hambre y el analfabetismo. Nunca el hombre había tenido una visión tan aguda sobre el sentido de la libertad, sin embargo surgen nuevos tipos de esclavitud social y psicológica en el mundo. Mientras el mundo es consciente de su unidad y de la dependencia que los une, está sin embargo dividido divido en direcciones opuestas. Hay divisiones en las esferas de la vida política, social, económica, racial e ideológica y sigue el peligro de una guerra que destruya todo.

Si bien aumenta el intercambio de ideas, el sentido mismo de las palabras que expresan conceptos de gran importancia, tienen diferentes sentidos en las distintas ideologías. Finalmente, se busca cuidadosamente el avance temporal, pero también avanzar en el crecimiento espiritual.

Por estas situaciones complejas, a muchos de nuestros contemporáneos se les impide reconocer verdaderamente los valores perennes y ubicarlos correctamente con los recientes 
descubrimientos. El género humano está en suspenso entre la esperanza y la angustia. Se hacen preguntas sobre el presente de las cosas. Este curso de las cosas obliga al hombre a responder por ellos. (Iglesia Católica, 1970, pp. 236-237).

\section{Hermenéutica de la categoría teológica de los "Signos de los tiempos"}

\section{La categoría teológica "Signos de los tiempos" en las Congregaciones Generales}

Lo primero que se ha de revisar es en cuáles de los cuatro esquemas que anteriormente se presentaron aparece esto de los "signos de los tiempos". En el texto de la $C^{11} 105$, se habla de que la Iglesia escruta, discierne los signos de los tiempos. Es más, ese es el título del entonces número 2. Pareciera ser que en el incipiente Esquema sobre la condición del ser humano en el mundo moderno la preocupación teológica por ver las señales de Dios en el presente constituye una tarea principal. Ahora bien, ¿cómo se realiza esta escucha? En CG 105 se sostiene que es un trabajo realizado desde la luz de la fe y desde el Espiritu Santo, con lo cual el discernimiento es eclesial, teológico y pneumatológico.

Un segundo elemento interesante es que en los cuatro textos, los Padres Conciliares presentan un 'diagnóstico' de la realidad en la que el mundo moderno se encuentra. Sin querer ser profetas de desventura, se afirma que mientras el género humano ha aumentado en la adquisición de conocimientos y de bienes materiales, por otro lado hay confusión, un sentimiento de vacío, desesperación y angustia. Entonces se pregunta: ¿cómo Dios está actuando en estas situaciones que claramente van en contra de la dignidad de la persona? ¿Son realmente signos de los tiempos? ¿Son voces del Espíritu? A pesar de las situaciones negativas, el Concilio tiene una palabra sobre la paz, la cual dice que nace de la caridad, la justicia, la verdad y la libertad. Pareciera ser que hacia allá quiere apuntar su servicio pastoral. Es entonces cuando tiene lugar la dimensión pneumatológica, por la cual se afirma que es el Espíritu el que renueva la faz de la tierra, purifica los corazones de las personas para optar por estos valores perennes (CG 161-166) y conduce finalmente al género humano hacia Cristo.

En el Esquema de la CG 132, se perciben algunas variaciones en las palabras y en la misma estructura del texto. El que antes fue el número 2 y estaba dentro del Proemio, ahora es el 4 y está dentro de la llamada Exposición introductoria. Los títulos también fueron modificados. Mientras que en la CG 105 el título era "La Iglesia escruta los signos

11. Sigla usada para hablar de las Congregaciones Generales.

12. González Carvajal (1987) a propósito de esto sostiene que "en el mismo Concilio se manifestó la tensión entre el sentido bíblico y el sentido sociológico de los signos de los tiempos y, por desgracia, ni siquiera allí venció siempre el primero. Debido a la presión de los biblistas, durante la reunión de Ariccia (31 de enero a 6 de febrero de 1965), la Comisión Redactora de Gaudium et Spes decidió sustituir en el número 4 la expresión "signos de los tiempos", que figuraba en el esquema de Zurich (1964) por la frase oportetcognoscere et intelligeremundum in quo vivimos, que ciertamente resultaba más precisa, puesto que lo que se hace a lo largo de toda la exposición preliminar de la Constitución no es buscar los signos de los tiempos actuales del Reinado de Dios, sino describir el mundo de hoy, con sus luces y sus sombras; es decir, tanto los signos del Reino de Dios como los signos del Reino del Mal” (pp. 27-28). 
de los tiempos", la CG 132 pasó a llamarse "Esperanzas y angustias", conceptos que son a nuestro entender los centrales en todo el documento de la Constitución Pastoral, al cual se puede sumar el tema de los gozos.

En este esquema no hay una mención explícita a los "Signos de los tiempos", o a las voces del Espíritu o a las señales de Dios. Más bien los Padres centran su atención en el diagnóstico que en CG 105 había sido más bien sucinto ${ }^{12}$. Pareciera ser que la intención de los Padres es trabajar en torno a un determinado método teológico, ya sea el ver-juzgar-actuar o del llamado método inductivo, el cual destaca el valor de lo particular, de lo histórico, de lo concreto y observable, situaciones que son iluminadas por los principios teológicos y pastorales.

El mundo moderno vive cambios y transformaciones sociales, económicas y políticas. Hay avances en cuanto a los bienes materiales y frente a ello hay más pobreza y analfabetismo. La distribución de esos mismos bienes no se realiza equitativamente. El ser humano, frente a estos cambios y transformaciones, tiene preguntas, siente temores y angustias, trata de responderse a las cuestiones más fundamentales, y es ahí donde la Iglesia debe tener alguna palabra fundada en el discernimiento, en este caso no explícito, de las señales de la presencia de Dios en la historia de la humanidad de este tiempo.

Ahora bien, la no mención explícita de los "Signos de los tiempos" como concepto, ¿significa que este ya no es un tema central para el Concilio? Si se revisan las Relaciones Particulares de la Exposición Introductoria de la CG 132, se lee que existe una subcomisión de los "signos de los tiempos" 13 la cual debe trabajar para recoger elementos universales que representen realidades densas y que puedan ser vistas como signos de los tiempos. Con esto aparece que se busca identificar situaciones, elementos, contextos que por su realidad signifiquen signos de la presencia de Dios.

En la Congregación General 161, aparecen los elementos nuevos en cursiva: "Es deber y tarea de la Iglesia el escrutar los signos de los tiempos a la luz del Evangelio. Por lo tanto y usando un lenguaje inteligible para cada generación, la Iglesia debe ser capaz de responder a los perennes interrogantes sobre la vida presente y la vida futura además de la relación entre ambas" (Iglesia Católica, 1970, p. 425) ${ }^{14}$. Con esto, se marca el querer propio del Concilio: escrutar los signos de los tiempos a la luz del Evangelio.

13. Castro (2011) nos cuenta que esta subcomisión estuvo presidida por Marcos McGrath, Obispo Auxiliar de la Arquidiócesis de Panamá y Decano de la Facultad de Teología de la UC desde el año 59' hasta el año 61'.Otros obispos pertenecientes a esta subcomisión eran Hélder Cámara (Brasil),Joseph Blomjous (Holanda) François Houtart (Bélgica), Karol Wojtyla (Polonia). McGrath, por ejemplo, sostenía que GS era un documento radicalmente nuevo y que por ello su método traía elementos que era necesario clarificar. Con esto se evidencia nuevamente la tensión teología - sociología propia de la futura Constitución Pastoral. La subcomisión de los signos de los tiempos se crea luego de la Reunión de Zurich en 1964. La otra comisión que nace es la teológica formada por Garrone, quien era su presidente, además de teólogos como Danielou, Congar y Rahner.

14. En la Relatio, aparece la explicación a este añadido (A). Esta nueva redacción responde a la petición del Obispo Franz Koning (Arzobispo de Viena; miembro de la Comisión Teológica del Concilio Vaticano II en su primer periodo) quien quiere subrayar: 1) La necesidad de que la Iglesia de siempre discierna los signos de los tiempos; 2) esta es la forma (este discernimiento) por medio de la cual conocemos el mundo de moderno. 
Ahora bien, ¿cómo se sirve al género humano de nuestro tiempo? Por medio de un lenguaje inteligible, es decir, se debe lograr una teología y una pastoral que sea significativa con el fin de poder adecuarse a las necesidades y perennes interrogantes de cada persona. El reconocimiento, diagnóstico o discernimiento, sostiene que el mundo posee expectativas y aspiraciones, pero que muchas de ellas están dominadas por el dramatismo. El género humano ha logrado poder económico, pero la distribución de los bienes materiales no llega a todos los pueblos.

El reconocimiento del Tercer Mundo y de su realidad sociopolítica y demográfica (pobreza y analfabetismo en palabras de Gaudium et Spes) ha sido también uno de los grandes méritos del Concilio, que es verdaderamente universal. Esto constituye una transformación social la cual ha llegado también a la vida de la fe y a la Iglesia ${ }^{15}$. La participación y el lugar de la Iglesia en el mundo es la clave interpretativa con la cual podemos entender qué ha significado el Concilio Vaticano II.

Siguiendo el tema del diagnóstico, la CG 161 sostiene que en el mundo moderno hay divisiones en las esferas política, social, económica, 'racial' e ideológica y sigue el peligro de una guerra (*) que destruya todo ${ }^{16}$. Estas divisiones, sigue el documento, impiden que el ser humano pueda reconocer verdaderamente los valores perennes de la historia y del Evangelio ${ }^{17}$. Entonces se formula la cuestión: ¿qué hace o debe hacer la Iglesia para unificar las miradas? Es ahí donde la Iglesia ha de servir a la persona por medio de un lenguaje inteligible y cercano, de manera que ayude al género humano a discernir cómo Dios actúa en estas realidades.

Finalmente, se revisará el texto de la Congregación General 166, el cual presenta un texto bastante parecido al de la Congregación General 161. En este texto aparece un elemento nuevo: los Modos ${ }^{18}$. Tres Padres dicen que la descripción es demasiado sociológica y retórica. En este primer momento se evidencia esta tensión entre teología y sociología de GS, texto magisterial eminentemente novedoso. Los modos también sostienen que hay pocas interpretaciones o mediaciones filosóficas: "Lo filosófico, lo religioso y el Evangelio se reducen a unas pocas líneas" (Tres Padres). Con esto se puede leer que la mediación más segura parece ser la filosófica.

El tema de las analíticas sociológicas ha sido siempre un tema 'conflictivo' para la teología, y ello se manifiesta, por ejemplo, en la Teología de la Liberación, a la cual se le ha criticado la falta del aparato filosófico. Parece ser que el tema de los "Signos de los

15. En la Relatiose explica el sentido de este añadido, a saber, que la transformación también llega a la vida de la fe y de la Iglesia. Esto fue solicitado por Paul Pierre Meouchi, patriarca de Antioquía de los Maronitas. En la Relatiose lee: "El Padre Meouchi pidió que se hablara también de transformación religiosa".

16. El tema de las divisiones en las distintas esferas del mundo actual (E), fue solicitado por Raúl Silva Henríquez, el cual pidió que se mencionara esta división de paradigmas viene desde el inicio de la humanidad.

17. El tema del no reconocimiento de los valores perennes de la historia, fue propuesta del Cardenal Kaczmarek cuando en la Relatio del número 4 del texto de la CG 161 se dice: "El Padre Kaczmarek dice que esto es causado por la privación del conocimiento de Dios, de la vida y de la vida futura. Esta es la razón de la adición".

18. Los Modos hacen referencia a los añadidos que los Padres Conciliares hicieron a los distintos esquemas. 
tiempos" aún está en esta disyuntiva de ser una mera interpretación socioanalítica con tintes marxistas, lo cual también fue dicho en los modos, cuando se lee que es mejor decir iluminación desde la Escritura y desde la fe que interpretación (En lugar de Evangelio, escríbase Sagradas Escrituras (Un Padre).

En lugar de 'interpretación' escríbase 'iluminador' (1 Padre), ya que puede provocarse la confusión de ser solo una mirada desde fuera, y no un trabajo eclesial. Con esto finalmente se puede evidenciar cómo la naciente teología de los "Signos de los tiempos" debía irse afianzando más ${ }^{19}$. Así, se puede entender que Gaudium et Spes no es un final, por el contrario, es un inicio.

\section{Comparación entre Humanae Salutis y \\ las Congregaciones Generales desde \\ los "Signos de los tiempos"}

Ahora bien, ¿cuál es la evolución de la categoría teológica "signos de los tiempos" desde Humanae Salutis hasta el esquema presentado en la CG 166? Se procederá a identificar brevemente una cierta 'estructura interna' en los cinco textos (Humanae Salutis y las Congregaciones Generales) de manera de vislumbrar cómo se van articulando y qué elementos permiten hablar de una continuidad o por el contrario de una ruptura. Humanae Salutis comienza exponiendo la doctrina cristológica por la cual Cristo aparece como el salvador del mundo. Esta dimensión no aparece en los textos de las CG, los cuales son más sociológicos que teológicos. Referencias cristológicas, por lo menos explícitamente no se evidencian.

El segundo elemento que presenta Humanae Salutis es lo eclesiológico, por lo cual se ve cómo la Iglesia es la portadora del Evangelio que debe ser anunciado a toda la creación, a la vez que la misma comunidad creyente realiza el discernimiento desde la fe y el Evangelio de los signos de los tiempos. El texto de la CG 105 manifesta abiertamente este último elemento (la Iglesia escruta, discierne los signos de los tiempos). Pareciera ser que el trabajo de la comisión teológica recoge el sentir de Juan XXIII de dar al Concilio un sentido de auscultar la realidad y mirar, a la luz de la fe, cómo en esa realidad hay presencia o no de la persona de Dios revelado en Jesucristo. El texto de CG 132 solo realiza el diagnóstico no nombrando la expresión "signos de los tiempos". Por su parte, CG 161 y 166 unen estos dos aspectos, a saber, el deber de la Iglesia de discernir las señales de los tiempos en medio de esta hora de transformaciones sociales, culturales y religiosas.

Junto con ello, la importancia del método de mirar la realidad, dinámica que fue ya había asumido Juan XXIII y que luego fue presentada como herméutica en los textos de la futura Gaudium et Spes. Finalmente, el sentido escatológico de los "signos de los tiempos" presentes en Humanae Salutis (en medio de tinieblas tan sombrías, percibimos numerosos indicios que parecen auspiciar un tiempo mejor para la humanidad y para la

19. A propósito del afianzar la teología de los "signos de los tiempos", Peter Hünermann sostiene que "hasta ahora no han sido elaborados criterios claros y universalmente válidos para la determinación de los signos de los tiempos, de manera que no se ha dado una utilización clara y sencilla de estos elementos importantes de la teología" (242). 
Iglesia) no está presente en los textos de las CG. Con esto, queda la sensación de que los cuatro textos pretenden ser una mirada más sociológica del sentido "signos de los tiempos" que una comprensión propiamente teológica. Esto fue lo que en los Modos del CG 166 se dice respecto que el texto es más 'sociológico'.

\section{Relación entre Gaudium et Spes 4 y 11}

Con lo que se ha revisado hasta ahora sobre el actual número 4 de GS, pareciera ser que este número posee un marcado acento sociológico, pero ¿dónde está lo propiamente teológico de la categoría "Signos de los tiempos"? Es ahí donde aparece otro texto que, a nuestro entender, debe leerse en conjunto con el que estamos estudiando. Nos referimos a GS 11, la cual tiene la característica de ser una exposición marcadamente teológica.

Mientras que GS 4 abordó el diagnóstico de la realidad histórica con todos su bemoles (género humano en un nuevo momento de la historia, cambios profundos y acelerados, técnica y creatividad humana, crisis de crecimiento, libertad, esclavitudes psicológicas y sociales, hambres, guerra, angustias y temores, preguntas por el sentido de la vida), GS 11 comienza exponiendo una teología que aborda las mociones del Espíritu a las cuales el Pueblo de Dios debe responder. Mientras el primer texto es de carácter ascendente (desde la historia hacia Dios), el segundo es descendente (la fe ilumina la realidad). En el segundo se encuentran expresiones tales como: "movimientos de fe", "Espíritu del Señor que llena el universo", "discernir en los acontecimientos los signos verdaderos de la presencia o de los planes de Dios", "fe que ilumina", "valores que nacen de la fuente divina y que son dados al hombre".

En estos dos textos (GS 4.11) acontece lo que constituye el corazón de la Constitución Pastoral sobre la Iglesia en el mundo actual, a saber, la relación necesaria entre teología y sociología, o entre el dato de la fe y las nuevas mediaciones culturales y humanas. La mirada sobre el mundo debe ser una que utilice las ciencias humanas para poder desentrañar sus imbricaciones, pero dicha mirada debe ser iluminada necesariamente por el dato revelado, por el Evangelio, por la experiencia eclesial, en definitiva, por la acción pneumatológica o la presencia permanente del Espíritu Santo.

La teología de los "Signos de los tiempos" es aquella que se desarrolla interdisciplinariamente, ya que busca dialogar con una realidad en constante transformación, que ayuda al ser humano por medio de lenguajes inteligibles y significativos por medio de los cuales pueda dar respuesta a sus temores y esperanzas, a sus gozos y tristezas (Cf. GS 1). Este documento del Concilio nos abre un camino, nos marca una dirección en nuestro ministerio teológico, sociológico, educativo. Los "signos de los tiempos" representan ese esfuerzo, esa intuición profética de Juan XXIII de poder mirar el mundo con los ojos del Verbo de Dios, ojos humanos y ojos divinos que iluminan nuestra historia, porque esa historia fue asumida radicalmente y a esa historia es enviada la Iglesia, la cual reconoce el mundo no como algo externo, sino como una nota esencial de su comunidad de creyentes. 


\section{El discernir los "signos de los tiempos" \\ como tarea irrenunciable para la Iglesia}

Al leer los anteriores textos del Concilio, se evidencia que hay una frase que se añade y que resulta ser el trabajo programático de la Iglesia del tiempo presente: deber de discernir los Signos de los tiempos, pero ¿por qué llamamos a esto una tarea irrenunciable? La importancia que el Concilio Vaticano II le da a la historia humana, valorándola en sus aspectos positivos y denunciando aquello que viola la dignidad de la persona, pasa también por la conciencia de que Dios sigue hablando en medio de los eventos históricos. ¿Cómo compatibilizar esto con la afirmación dogmática que dice que la revelación finaliza con la muerte del último apóstol? ¿son realmente palabras nuevas? ¿o son más bien herramientas interpretativas, señales, llamados a la conciencia eclesial para asumir un nuevo tiempo?

La teología de los "Signos de los tiempos" significa que la Iglesia está en el mundo. Acontece, por un tanto un resituamiento de la comunidad creyente que busca comprender que la historia representa un lugar teológico, en el cual Dios se está manifestando. El Verbo de Dios desde el momento en que se encarna en la cultura del ser humano, los acontecimientos seculares (la cultura, la política, los Derechos Humanos, la educación, etc.) llevan en sí elementos que, como dijo Juan XXIII en Humanae Salutis, permite vislumbrar que en medio de las tinieblas, de las angustias, de las sensaciones de vacío, existen momentos históricamente densos y particularmente significativos, en los cuales acontece alguna palabra de Dios o una moción del Espíritu. En el mundo y en la historia está encarnada la Iglesia. Ella no puede desinteresarse del mundo y por ello el discernir los signos de los tiempos, como mandato de Jesús (Mt 16,3), representa una tarea irrenunciable, ya que solo así podrá cumplir el cometido que el mismo Concilio se propuso, a saber, servir a la totalidad del género humano, de manera que acomodándose a cada generación y por medio de un lenguaje claro pueda responder a las siempre presentes interrogantes del género humano.

\section{Breve mirada latinoamericana}

a los Signos de los tiempos

Aunque el motivo del artículo presente era estudiar el proceso redaccional de la categoría teológica en las Actas del Concilio Vaticano II que dieron lugar a la posterior Constitución Pastoral Gaudium et Spes, ha parecido necesario presentar un breve apartado sobre cuál es la comprensión y discusión teológica que en Latinoamérica se da sobre los Signos de los tiempos. Para efectos de esta parte del artículo, se presentará la reflexión que el documento magisterial más reciente del Episcopado Latinoamericano y del Caribe, el texto de Aparecida ${ }^{20}$, aborda en cuanto a la temática de los "Signos de los tiempos".

20. De ahora en adelante DA. Nos referimos a la quinta conferencia del Episcopado Latinoamericano celebrada en el Santuario Mariano de Aparecida, Brasil, el año 2007. El entonces cardenal Jorge Bergoglio, actualmente Papa Francisco, fue en gran medida el responsable de la elaboración del documento final. 
El documento de Aparecida

y los "signos de los tiempos"

La relevancia de la teología del documento de Aparecida (DA), está radicada en revitalizar la evangelización y la misión de las comunidades cristianas de base, especialmente en aquellos lugares que representan discontinuidad, y en los cuales se ubican los despreciados por las estructuras económicas, pedagógicas, políticas o ideológicas. La reflexión proveniente de América Latina, constituye una revolución en el orden de las teologías, ya que constituye una reflexión netamente continental, en la que se presenta la praxis de liberación de los más pobres. Esta teología surge posterior al Vaticano II, acontecimiento que provoca una renovación en la forma de concebir a la Iglesia y de cómo ella presenta su práctica pastoral.

Desde la perspectiva del discipulado y de la misión que Jesús propone, que es la gran eclesiología del DA, uno de los elementos claves es el discernimiento y la escucha de los signos de los tiempos, comprendidos como elementos históricos en los cuales podemos entrever la acción de Dios. Por medio del discernimiento, la comunidad va ejerciendo su praxis de liberación integral, especialmente con los más pobres, que son por los que en definitiva Dios opta y los cuales se conciben como lugares teológicos, es decir, mediaciones históricas por medio de las cuales Dios nos interpela.

El sentido que el documento de Aparecida otorga a los signos de los tiempos, está en la misma línea de la reflexión conciliar, ya que se sostiene que dichas señales se presentan en la comunidad desde "una realidad marcada por grandes cambios que afectan profundamente sus vidas" (CELAM, 2007, N. ${ }^{\circ} 33$ ) Con ello, se nos impone comprender el contexto histórico de nuestro continente, de manera de buscar los signos de la presencia de Dios. La situación histórica que se establece como escenario para dicha búsqueda, "se ha vuelto para el ser humano cada vez más opaca y compleja" (CELAM, 2007, N. ${ }^{\circ}$ 36), esto a causa de la presencia de estructuras de pecado que van deshumanizando al ser humano y violentando su dignidad de ser imagen de Dios. Pese a esta realidad, aún se presentan las señales de la presencia de lo divino, las cuales necesitan ser escuchadas de manera atenta y de esta manera discernir "lo que el Espíritu está diciendo a las Iglesias" (Ap 2, 29) a través de los signos de los tiempos en los que Dios se manifiesta (CELAM, 2007, N. $\left.{ }^{\circ} 366\right)$

La hermenéutica que se debe ejercer en el discernimiento de la acción de Dios en la vida social, debe ser una que comporte el "ponernos al servicio del Reino, anunciado por Jesús, que vino para que todos tengan vida y "para que la tengan en plenitud" (Jn 10, 10)" (CELAM, 2007, N. ${ }^{\circ} 33$ ) Por medio del ejercicio de la acción misionera y evangelizadora, especialmente con los más pobres, estamos leyendo de buena manera la presencia de lo trascendente en lo secular, y estamos provocando el que nuestros pueblos lleguen a la vida abundante de Jesucristo.

Un aspecto constitutivo de los "Signos de los tiempos", radica en la valorización de la historia. Con esto se sostiene que las realidades sociales no pueden ser ajenas al actuar de la Iglesia, esto porque ella se encuentra encarnada en el mundo, con lo cual está llamada a responder a las constantes preguntas por el sentido de la existencia humana. Una Iglesia que se mantenga fuera de las vicisitudes temporales, muy difícilmente podrá 
cumplir el cometido mandado por Jesucristo. Debe por lo tanto, producirse una constante inculturación, con la cual "la Iglesia se enriquece con nuevas expresiones y valores, manifestando y celebrando cada vez mejor el misterio de Cristo" (CELAM, 2007, N. ${ }^{\circ} 479$ )

La preocupación de la Iglesia por ayudar a despertar el sentido misionero de las comunidades, responde a la urgencia que se presenta en el anuncio del Evangelio de liberación estableciendo así "la presencia de los valores del Reino de Dios en las culturas, recreándolas desde dentro para transformar las situaciones antievangélicas" (CELAM, $\left.2007, n^{\circ} 74\right)$. Con esto, se podrán establecer auténticas praxis mesiánicas especialmente con los más marginados de los sistemas, las cuales se constituyen como signos de los tiempos, pero esto será analizado en el siguiente punto.

Situación de los pobres como lugar teológico y

los "Signos de los tiempos" desde Aparecida

La importancia de las mediaciones históricas por las cuales se provoca el encuentro de la comunidad creyente con Jesucristo, son expuestas por Aparecida, comenzando desde las más clásicas (oración, Eucaristía, Lectio Divina, Liturgia o los sacramentos), hasta los nuevos lugares de encuentro. En estos últimos encontramos a los pobres, los cuales son comprendidos por la teología latinoamericana como la concreción histórica de Cristo crucificado. Nos dice Aparecida que a Jesús "también lo encontramos de un modo especial en los pobres, afligidos y enfermos (cf. Mt 25, 3740), que reclaman nuestro compromiso y nos dan testimonio de fe, paciencia en el sufrimiento y constante lucha para seguir viviendo" (CELAM, 2007, N. ${ }^{\circ} 257$ ).

Nuestra realidad latinoamericana se presenta como una netamente oprimida por ideologías o sistemas, los cuales tienen como sus principales víctimas a los pobres. Ellos toman variados rostros, entre los que encontramos a las comunidades indígenas y afroamericanas, a las mujeres víctimas de la violencia, a los jóvenes que reciben una educación de baja calidad, a desempleados, migrantes, desplazados, campesinos, a los niños, a los no nacidos, entre muchos otros (Cf. CELAM, 2007, n65) En estos rostros, el Verbo Encarnado se revela de manera oculta, y desde estas mediaciones nos interpela para generar prácticas de liberación integral.

La opción que la Iglesia latinoamericana realiza por estos hermanos, "es fundamentalmente una dimensión constitutiva de nuestra fe en Jesucristo" (CELAM, 2007, n²57) En virtud de esta fe, se requiere que "discípulos de nuestras Iglesias vayan y evangelicen en la "otra orilla" (CELAM, 2007, N. ${ }^{\circ} 379$ ), en las que radican los lugares de discontinuidad, en los cuales encontramos a Jesús encarnado históricamente en los pobres. Hacer la opción de Jesucristo y estar con los marginados, participar en sus luchas de liberación, desde la fe y sostenerse por la palabra interpretada desde la comunidad, constituye un signo de los tiempos, que en la teología latinoamericana se concibe como la "opción preferencial por los pobres". Dicha opción es reconocida por Aparecida como algo que se encuentra de manera "implícita en la fe cristológica en aquel Dios que se ha hecho pobre por nosotros, para enriquecernos con su pobreza" (CELAM, 2007, N. ${ }^{\text {o } 392) . ~}$

Es necesario que la comunidad creyente se disponga a cruzar a la orilla de los desheredados del mundo, lugar en el cual se les abrirán los ojos y podrán comprender la 
realidad y la novedad del Dios y de su Hijo Jesucristo, hecho hermano nuestro por la Encarnación. La revolución que presenta el Reino, radica justamente en hacer de los pobres los protagonistas de la nueva sociedad. A ellos se les debe anunciar el Evangelio, en el cual se contienen los valores mesiánicos de "dignidad, justicia, paz, fraternidad, sacrificio, cuidado de la creación, existencia por otros, libertad" (Casale, 2011, p. 39). Por tanto, la Palabra de Dios debe continuar siendo proclamada como anuncio gozoso de la libertad que ya llega en Jesucristo, a la cual la Iglesia debe fidelidad desde su acción misionera.

\section{Síntesis ¿Qué son finalmente los "Signos de los tiempos"?}

Al finalizar el presente desarrollo, que a nuestro entender constituye un pre-texto para seguir reflexionando y pensando la Iglesia después de 50 años de finalización del Concilio y de la promulgación de Gaudium et Spes, se procederá a intentar una respuesta a la interrogante de qué son finalmente los "Signos de los tiempos" desde la presentación de algunas características que esta categoría teológica presenta en los textos de GS 4, 11 y 44 .

1. Los "Signos de los tiempos" y su discernimiento representan un esfuerzo para cumplir la misión que la Iglesia tiene de servir al ser humano: "El Concilio, testigo y expositor de la fe de todo el Pueblo de Dios congregado por Cristo no puede dar prueba mayor de solidaridad, respeto y amor a toda la familia humana que la de dialogar con ella (con la familia humana) acerca de todos estos problemas, aclarárselo a la luz del Evangelio y poner a su disposición del género humano, el poder salvador que la Iglesia, conducida por Espíritu Santo, ha recibido de su Fundador" (Pablo VI, 1971, parágrafo 3).

2. Los "Signos de los tiempos" son signos de este periodo nuevo de la historia que está caracterizada por cambios profundos y acelerados que abarcan el mundo entero (Cf. GS 4) y voces de nuestro tiempo (GS 44). Estos cambios han sido provocados por el ser humano con su inteligencia y con el aumento del poder sobre la técnica. Todo confluye en que se está en presencia de una metamorfosis social, cultural y religiosa (Cf. GS 4).

3. Los "Signos de los tiempos" son experiencias de la familia humana en las que se mezclan tanto las esperanzas, las aspiraciones y el dramatismo (Cf. GS 4). Son también los acontecimientos, las exigencias y los deseos en los que la Iglesia participa junto con sus contemporáneos (Cf. GS 11).

4. En los "Signos de los tiempos" se encuentra la presencia de Dios o sus planes (Cf. GS 11), experiencias de fe que es necesario discernirlas desde la fe, la cual es animada por el Espíritu Santo (Cf. GS 11). Dicha fe es una luz que permite comprender cuál es la vocación última de la persona, con lo cual el juicio de la época hecho en clave teológica posee un alto componente antropológico y pneumatológico. El propósito antropológico del Concilio es volver a unir al ser humano con Dios desde la consideración de la búsqueda de los valores perennes los cuales han de ser compaginados de buena manera con los nuevos descubrimientos (Cf. GS 4). La técnica así está llamada a la humanización. 
5. La teología de los "Signos de los tiempos" constituye una mirada positiva que la Iglesia hace de la realidad, mirada que se realiza desde la esperanza (escatología) de que después de las tinieblas que parecen sumergir a la presente época vendrán tiempos mejores para el mundo y para la Iglesia (Cf. Humanae Salutis). En definitiva los "Signos de los tiempos" es un mirar y un escuchar bien reconociendo el paso del Señor por la única historia.

Pueden existir muchas otras características, pero siguiendo los planteamientos teológicos de varios pensadores, la teología de los "Signos de los tiempos" representa finalmente un esfuerzo naciente de una Iglesia que se siente solidaria con el mundo, de la Iglesia que está en salida, que mira la realidad con los ojos de Dios, discernimiento que le viene como un movimiento de fe y no solo de analítica sociológica. Poner en diálogo las ciencias humanas desde el inicio de la fe significará finalmente asumir los desafíos, las voces de nuestros contemporáneos, sus deseos, angustias, esperanzas y proyectos comunes, en los cuales se debe reconocer al Verbo de Dios que se ha unido de cierta manera con todo el género humano (Cf. GS 22).

\title{
ABSTRACT
}

\section{"Signs of the Times" in GaudiumetSpes: Writing, Hermeneutics and Theology}

\begin{abstract}
The following article is about a brief compositional and hermeneutic study of the theological categories of "Signs of the Times" present in the Gaudium et Spes Pastoral Constitution of the Vatican Council II, and in the General Congregations prepared with this document. To study "Signs of Time" theology requires that the believer be able to accept the history and the culture he lives in, the presence of God may be found in order to invite new paths of act and ecclesial renovation.
\end{abstract}

Key words: Signs of time, GaudiumetSpes, General Congregations, Vatican Council II, history

\section{RÉSUMÉ}

\section{"Signes des temps" dans Gaudium et Spes:}

Rédaction, herméneutique et théologie

L'article suivant rend compte d'une courte étude d'ordre rédactionnel et herméneutique de la catégorie théologique "Signes des temps » présent dans la Constitution Pastorale Gaudium et Spes du Concile Vatican II et dans les Congrégations Générales qui ont préparé ce document. Étudier la théologie de Signes des temps exige que le croyant puisse assumer que dans l'histoire et dans la culture dans laquelle il vit et cohabite, il est possible de trouver la présence de Dieu pour susciter de nouveaux chemins d'actions et de renouvellement ecclésial.

Mots-clés:Signes des temps, Gaudium et Spes, Congrégations générales, Concile Vatican II, histoire.

\section{Referencias Bibliográficas}

Bausenhart, G. (2012). De la esencia de la Iglesia a su identidad: El cambio de paradigma eclesiológico en el Concilio Vaticano II. Palabra y Razón, diciembre vol 2, 9-36. 
Bentue, A. (2001).Espíritu de Dios y espiritualidad laical. San Pablo: Chile.

Castro, F. (2011).Cristo y cada hombre: Hermenéutica y recepción de una enseñanza del Concilio Vaticano II. GregorianBiblicalPress: Roma.

Casale, C.(2011). Discernir la acción de Dios en la historia. Revista Mensaje, 601, 357-361.

CELAM. (2007). Documento Conclusivo V Conferencia General del Episcopado Latinoamericano y del Caribe. San Pablo: Chile.

González-Carvajal, L. (1987). Los signos de los tiempos, El Reino de Dios está entre nosotros. Sal Terrae: Santander.

Hünermann, P. (2014). Vaticano II como software de la Iglesia actual. Universidad Alberto Hurtado: Santiago de Chile.

Iglesia Católica (1970). Acta synodaliasacrosanctiConciliiOecumeniciVaticani II. TypispolyglottisVaticanis: Ciudad del Vaticano.

Juan XXIII. (1971). Concilio Vaticano II. Constitución Humanae Salutis. 25 de diciembre de 1961. En Iglesia Católica. Documentos del Concilio Vaticano II. Biblioteca de Autores Cristianos: Madrid.

Noemi, J. (2007). En la búsqueda de una teología de los "signos de los tiempos". Teología y Vida, Vol. XLVIII, 439-447.

Pablo VI. (1971). Concilio Vaticano II. Constitución Pastoral Gaudium et Spes. 7 de diciembre de 1965. En Iglesia Católica.Documentos del Concilio Vaticano II. Biblioteca de Autores Cristianos: Madrid. 